\title{
Monocyte Chemoattractant Protein-1 in Antineutrophil Cytoplasmic Autoantibody-Associated Vasculitis: Biomarker Potential and Association with Polymorphisms in the MCP-1 and the CC Chemokine Receptor-2 Gene
}

\author{
Nina Jönsson, Evelina Erlandsson, Lena Gunnarsson, Åsa Pettersson, and Sophie Ohlsson (iD \\ Department of Nephrology, Institution of Clinical Sciences in Lund, Lund University, Lund, Sweden \\ Correspondence should be addressed to Sophie Ohlsson; sophie.ohlsson@med.lu.se
}

Received 8 September 2017; Accepted 22 January 2018; Published 12 March 2018

Academic Editor: Yona Keisari

Copyright (C) 2018 Nina Jönsson et al. This is an open access article distributed under the Creative Commons Attribution License, which permits unrestricted use, distribution, and reproduction in any medium, provided the original work is properly cited.

\begin{abstract}
Antineutrophil cytoplasmic autoantibody- (ANCA-) associated vasculitis (AAV) are relapsing-remitting disorders with unpredictable prognosis. There is a need of biomarkers for distinguishing which patients will have a more severe outcome and also for predicting relapses in disease activity. This study confirms the previous results of urinary MCP-1 (uMCP-1) as a prognostic marker and explores its potential as a marker of disease activity. Method. 114 patients with AAV were followed regularly between 2002 and 2011 at Skåne University Hospital. Urine samples, blood samples, and clinical status were registered. The urine samples were analyzed in an in-house-developed ELISA. PCR-RLFP was used to analyze the MCP-1 and CCR2 genes. Results. Patients with severe prognosis had significantly higher levels of uMCP-1 compared to patients with nonsevere prognosis and healthy controls. Patients with renal damage had higher levels compared to patients who did not have renal damage. There was also a tendency of higher uMCP-1 levels in active disease as compared to remission. AA in the -2518 position in the MCP-1 gene was associated with a more severe outcome compared to the A/G or the G/G genotype. The A/A genotype were also associated with higher levels of uMCP-1. No significant associations were seen for the CCR2-V64I. Conclusion. This study confirmed the connection between high uMCP-1 levels and poor prognosis and also disease activity. It also suggests an association of the A/A genotype at position -2518 in the MCP-1 gene and poor prognosis in AAV. uMCP-1 is clearly a candidate biomarker of potential clinical value. The A/A genotype association needs further evaluation.
\end{abstract}

\section{Introduction}

The antineutrophil cytoplasmic autoantibodies (ANCA) were discovered in 1982 in 8 patients with segmental necrotizing glomerulonephritis [1]. Major targets for ANCA are myeloperoxidase (MPO) and proteinase 3 (PR3) in monocytes and neutrophils [2]. ANCA is strongly associated with three types of small vessel vasculitis: microscopic polyangiitis (MPA), granulomatosis with polyangiitis (GPA), and eosinophil granulomatosis with polyangiitis (EGPA) [3].

Most patients with GPA have ANCA against PR3 and most patients with MPA have ANCA against MPO, whereas in EGPA, the majority is ANCA negative although MPO-
ANCA occur. In systemic GPA, ANCA positivity is seen in more than $90 \%[3,4]$. About $10 \%$ of patients with GPA or MPA have negative assay for ANCA, especially those with less severe disease $[5,6]$.

ANCA is capable of activating the leukocytes to adhere to the endothelial cells in the vessel wall and of inducing an inflammatory process. It is thereby thought to play a role in the pathogenesis of small vessel vasculitis [7, 8]. Patients with ANCA against PR3 have been shown to have more frequent respiratory tract granulomas and extra renal organ manifestations compared to patients with ANCA against MPO. Anti-PR3 ANCA has also been seen to correlate with a faster decline in renal function and more frequent relapses compared to anti-MPO-ANCA [9]. 
ANCA-associated vasculitis affects mainly capillaries, and also arterioles and venules, and in some cases middlesized blood vessels. AAV is most common in adults in their '50s and '60s. The distribution among men and women is equal [5]. Prevalence in the healthcare district around the city of Lund in southern Sweden per million inhabitants is 94 for GPA, 31 for MPA, and 14 for EGPA, which is the highest prevalence reported in the world so far [10]. The 5-year survival for GPA is $74-91 \%$, for MPA $45-76 \%$, and for EGPA 60-97\% [11].

Cyclophosphamide and glucocorticoids have been used as treatment in AAV for over 40 years, and the combination is the standard treatment for remission induction. If the patient has normal renal function and a less severe disease, a combination of methotrexate and glucocorticoids can be used instead as a less toxic option. To maintain remission, low doses of glucocorticoid and either methotrexate, azathioprine, or sometimes mycophenolate mofetil are recommended. Other treatment such as immunoglobulin or rituximab can be considered as alternative treatment in relapsing, refractory, or persistent disease [12]. Treatment with cyclophosphamide is associated with morbidity and mortality due to myelosuppression, infection, and malignancy [13]. Mortality due to ANCA-associated vasculitis and immunosuppressive treatment has however decreased over the last 4 decades, as dose regimens have been optimized [14].

To further optimize and individualize treatment, there is a need for biomarkers that can predict poor outcome. Renal function at diagnosis is a strong predictor of patient survival [15-17]. Other factors shown to correlate with poor prognosis are IL-8 [18], IgM [19], and high levels of PR3-ANCA measured by capture ELISA [20]. In a previous study, uMCP-1 was shown to be able to predict poor prognosis and the association was stronger not only compared to BVAS, CRP, and ANCA, which are markers used today to follow disease activity, but also compared to new markers shown to be able to predict poor prognosis such as urinary IgM and IL-8 [21].

There is also a need for biomarkers that are able to predict relapse. ANCA levels have been shown to predict relapses in GPA, but the relapses often occur more than 6 months after a rise in ANCA levels and the sensitivity and specificity are not very high [22]. A proteomics study from 2009 describes a number of biomarkers able to distinguish between patients with active AAV and patients in remission. Proteolytic products of hemoglobin were the most frequently observed, but fragments of albumin and $\alpha 1$-antitrypsine were also seen [23].

MCP-1, also called chemokine (C-C motif) ligand 2 (CCL2), was the first CC chemokine to be discovered. It is composed of 76 amino acids and the gene is located on chromosome 17. MCP-1 is produced by endothelial, epithelial, smooth muscle, mesangial, astrocytic, monocyte, and microglial cells as well as fibroblasts. Monocytes/macrophages are the major source, and MCP-1 is a potent chemotactic factor for monocytes. uMCP-1 has been shown to be useful as a marker in diseases such as systemic lupus erythematosus (SLE) and diabetes mellitus [24, 25].
Single-nucleotide polymorphism (SNP) in the gene regulatory region at position $-2518 \mathrm{~A} / \mathrm{G}$ in the MCP-1 gene have been reported. Healthy individuals with the $\mathrm{A} / \mathrm{G}$ or $\mathrm{G} / \mathrm{G}$ genotype are producing more MCP-1 than individuals homozygous for the A/A genotype [26]. However, another study showed that the A/A genotype was associated with renal disease in SLE and elevated levels of MCP-1 in both serum and urine [27]. The GA and AA genotype is associated with clinical severity in Behcet's disease [28]. The GG genotype is associated with higher risk of chronic obstructive pulmonary disease (COPD) [29], oral squamous cell carcinoma [30], and late acute rejection in kidney-transplanted patients [31].

CCR2 is mainly expressed on monocytes and is the receptor not only for MCP-1 but also for MCP-2, MCP-3, and MCP-4. It has 374 amino acids and the aminoterminal domain is necessary for binding of MCP-1 [32]. In CCR2 polymorphism at position 190 from $\mathrm{G}$ to $\mathrm{A}$, a mutation called CCR2-V64I causes a change from valine to isoleucine at codon 64, and it has been shown that this change causes a higher expression of CCR2A compared to CCR2B, which in turn downregulates the cell surface expression of CCR5 [33].

It has been shown that this polymorphism is protective in the development and progression not only of inflammatory diseases like multiple sclerosis (MS) [34] and acquired immunodeficiency syndrome (AIDS) [35] but also of malignancies such as breast cancer [36].

The aim of this study was to further establish the correlation of uMCP-1 levels and poor prognosis shown previously [21]. We also wanted to explore the connection between uMCP-1 levels and disease activity. In addition, the -2518 A/G polymorphism in the MCP-1 gene and the CCR2V64I polymorphism in the CCR2 gene has been studied.

\section{Materials and Methods}

2.1. Patients. Patients who visited the Department of Nephrology at Skåne University Hospital between 2002 and 2010 with an ANCA-associated vasculitis (AAV) diagnosed according to the Chapel Hill criteria were asked to participate in the study. Classification of AAV was done according to the consensus methodology described by Watts et al. in 2007 [37]. Patients on dialysis and patients with cancer were not included. The patients were followed by regular visits at the open patient clinic between 2002 and 2011. Each time, blood and urine samples were taken and clinical status was registered. Clinical status was measured using the Birmingham vasculitis activity score (BVAS) [38] and development of critical damage was according to the vasculitis damage index (VDI) [39]. Clinical status was classified as remission (BVAS $0-1$ ), chronic grumbling activity (BVAS 2-5), or relapse/new disease activity (BVAS $>6$ ). The patient was considered having a severe prognosis if the patient fulfilled at least one of the following criteria: (1) chronic kidney failure with glomerular filtration rate (GFR) < 30, (2) stroke after diagnosis, (3) myocardial infarction after diagnosis, (4) subglottic stenosis, (5) respiratory insufficiency requiring oxygen treatment, (6) start of dialysis treatment or kidney transplantation after diagnosis, and (7) death. Patients who did not meet any of these 
TABle 1: Characteristics of patients included in the study. Patients with AAV followed at SUS, Lund, 2002-2011.

\begin{tabular}{lc}
\hline Number of patients & 113 \\
Number of urine samples & 670 \\
Urine samples per patient, median (range) & $5(1-22)$ \\
Proportion women & $50.0 \%(n=57)$ \\
Age at last follow-up, years (range) & $62.6(21-91)$ \\
Deceased at last follow-up & 23 \\
Diagnosis & \\
Diagnosis confirmed with renal PAD & 62 \\
GPA & 74 \\
MPA & 39 \\
ANCA specificity & \\
MPO & 40 \\
PR3 & 68 \\
PR3 + MPO & 1 \\
Negative & 3 \\
\hline
\end{tabular}

AAV: antineutrophil cytoplasmic autoantibodies- (ANCA-) associated vasculitis; PAD: pathologic anatomic diagnosis; GPA: granulomatosis with polyangitis; MPA: microscopic polyangitis; MPO: myeloperoxidase; PR3: proteinase 3.

criteria were classified as having a nonsevere prognosis. The control group consisted of healthy blood donors. Urine samples were also collected from 9 patients with other vasculitis diagnoses. All patients and controls gave their written informed consent to participate, and the study was accomplished with permission from the local ethical committee of Lund (see Table 1 for more patient characteristics).

2.2. Blood and Urine Samples. Urine samples of first-voided urine were collected in polyethylene vessels (Kebo $A B$, Sweden). The urine samples were kept frozen at $-20^{\circ} \mathrm{C}$ until assayed. Blood samples were collected in EDTA tubes and centrifuged, and the plasma was stored in $-20^{\circ} \mathrm{C}$.

2.3. MCP-1 Enzyme-Linked Immunosorbent Assay (ELISA). Human monocytes were isolated from healthy donors using OptiPrep as described by the manufacturer. From the monocytes, total cellular mRNA was isolated using Qiagen mRNA purification kit as described by the manufacturer. cDNA was obtained by one-step reverse transcription polymerase chain reaction (RT-PCR), using a forward oligonucleotide primer (primer D04 F: GAAACTATTTTATCAAAAGCATGC) and a reverse oligonucleotide primer (primer D05 R: GGCAATTATCATAGCCAGCAG). RNAse inhibitor, onestep enzyme reverse transcriptase and deoxyribonucleotide triphosphates (dNTPs) were used during the PCR. The PCR product from the PCR was analyzed on a $1 \%$ agarose gel, and MCP-1 (300 bp) was extracted by using MinElute Gel Extraction Kit (Qiagen) as described by the manufacturer. cDNA was cloned into a pCR2.1TOPO (TA $3.9 \mathrm{~kb}$ ) vector using (TA Cloning Kit, Invitrogen) as described by the manufacturer.

Expression and purification of MCP-1 was performed as previously described [40]. Instead of insect cells, human

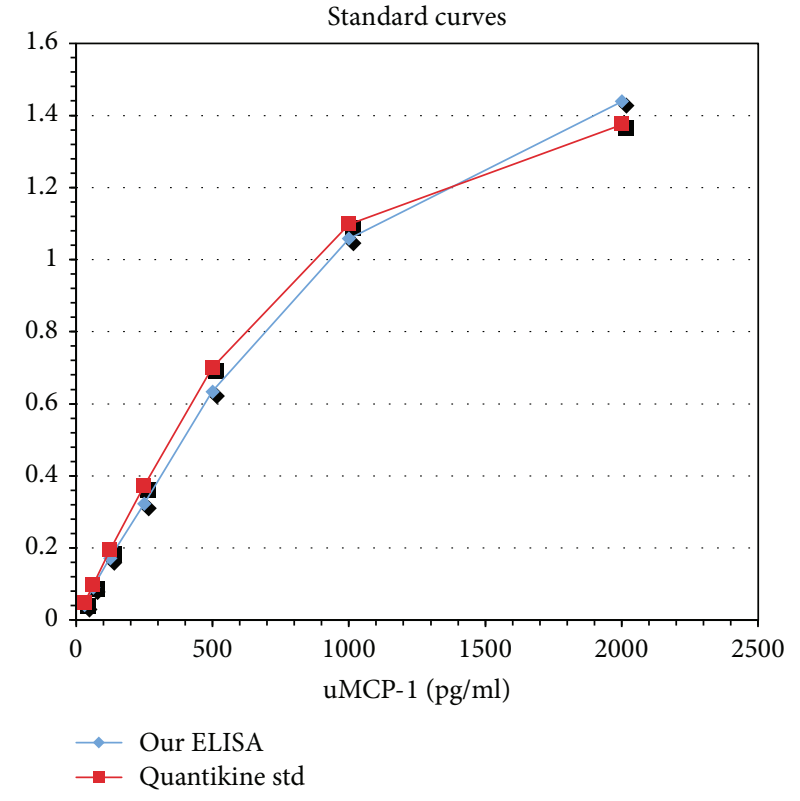

Figure 1: Comparison of the in-house-developed ELISA and the commercial ELISA kit. The graph shows the comparison of ELISA's ability to measure determined levels of added MCP-1, the standard curve in the ELISA. Absorbance at $405 \mathrm{~nm}$, background absorbance subtracted. ELISA: enzyme-linked immunosorbent assay; uMCP-1: urinary monocyte-chemoattractant protein-1.

embryonic kidney (HEK) 293 cells were used. These were cultured in DMEM with $10 \%$ fetal calf serum (FCS). Transfection with the plasmid containing the MCP-1 gene was made by electroporation. Selection of the transfected cells was performed using geneticin. Recombinant MCP-1 was purified in two steps. First, the supernatant of the collected serum-free cell medium was added directly on a Mono $\mathrm{S}$ HR 55 column. The bound protein was eluted with a $50 \mathrm{~min}$ linear gradient from 0 to $1 \mathrm{M} \mathrm{NaCl}$ in $20 \mathrm{mM}$ MOPS, $\mathrm{pH}$ 6.5. Fractions were collected and to verify MCP-1 content, a capture ELISA using rabbit anti-MCP-1 (Abcam) was used. The fractions containing MCP-1 were assayed in a Western blot to explore the purity using rabbit anti-MCP1 (Abcam). Fractions containing MCP-1 were pooled. To further purify MCP-1, the fraction was gel filtered on a Superdex 75 HR 1030 column in $0.1 \mathrm{M}$ ammonium acetate. Again, a Western blot and a capture ELISA were performed to find the fractions containing MCP-1, which were pooled. The collected MCP-1 was sent to Innovagen for immunization of rabbits resulting in rabbit anti-MCP-1. Innovagen also biotinylated some of the rabbit anti-MCP-1.

The ELISA was developed analyzing urine samples with both Quantikine and our own antibodies from Innovagen. The standard curves were similar (see Figure 1). The results from the in-house-developed ELISA had a mean variation of $9.0 \%$ compared to the Quantikine kit. A recovery test was made adding 10, 100, and $200 \mathrm{pg} / \mathrm{ml}$ Quantikine MCP1 to four test urine samples with a mean variation of $12.9 \%$. Inter- and intraassay tests were made with a mean variation of 6 and $10 \%$. 
Microplates were precoated with rabbit anti-MCP-1 and left in $+8^{\circ} \mathrm{C}$ overnight. The urine samples were diluted $1: 2$ in Tris buffer $\left(50 \mathrm{mM}\right.$ Tris $\mathrm{HCl}, 0.05 \% \mathrm{NaN}_{3}, 0.2 \%$ bovine serum albumin (BSA), and $0.05 \%$ Tween 20 ) and then added to the microplates and left to incubate for two hours in room temperature. Biotinylated rabbit anti-MCP-1 was used as a secondary antibody and left to incubate for two hours in room temperature. After that, streptavidin-AP was added to each well and left to incubate for one hour in room temperature. A substrate was added and the absorbance was read after $45 \mathrm{~min}$ incubation in room temperature. Between every step, the microplates were washed three times in washing solution $(0.9 \% \mathrm{NaCl}, 0.05 \%$ Tween $)$.

2.4. Laboratory Work-Up. Urine samples were sent to the Clinical Chemistry Department at Skåne University Hospital for measurement of the creatinine levels and urine albumin/ creatinine index. Blood samples were sent for analysis of C-reactive protein (CRP), white blood cell count, creatinine, and cystatin C. PR3-ANCA and MPO-ANCA were analyzed at Wieslab, Euro Diagnostica, Malmö.

2.5. MCP-1 Polymorphism. The MCP-1 -2518 A/G polymorphism was genotyped using restriction fragment length polymorphism polymerase chain reaction (PCR-RLFP) as previously described [29]. Genomic DNA was extracted from peripheral blood by using AllPrep DNA/RNA Mini Kit (Qiagen) according to the manufacturer's protocol. $0.5 \mu \mathrm{l}$ forward primer $5^{\prime}$-TCTCTCACGCCAGCACTGACC-3' and $0.5 \mu \mathrm{l}$ reverse primer $5^{\prime}$-GAGTGTTCACATAGGC TTCTG-3' (Invitrogen) were added to the DNA together with $6 \mu \mathrm{l} \mathrm{MgCl}_{2}, 1 \mu \mathrm{l}$ dNTPs, $2 \mu \mathrm{l}$ Taq polymerase, $\mathrm{H}_{2} \mathrm{O}$, and $5 \mu \mathrm{l}$ buffer to a total volume of $50 \mu \mathrm{l}$. Amplification occurred by $5 \mathrm{~min}$ denaturation in $94^{\circ} \mathrm{C}$ and thereafter 30 cycles of denaturation for $1 \mathrm{~min}$ in $94^{\circ} \mathrm{C}$, annealing for $1 \mathrm{~min}$ in $55^{\circ} \mathrm{C}$, and extension for $1 \mathrm{~min} 30 \mathrm{~s}$ in $72^{\circ} \mathrm{C}$. The process ended with $7 \mathrm{~min}$ extension in $72^{\circ} \mathrm{C}$. The PCR products were digested using Pull (Fermenta) at $37^{\circ} \mathrm{C}$ overnight. MCP-1 -2518 G/A variants were detected by electrophoresis on $3 \%$ agarose gel.

2.6. CCR2 Polymorphism. Genomic DNA was extracted using AllPrep DNA/RNA Mini Kit (Qiagen). Forward primer $5^{\prime}$-TTGTGGGCAACATGATGG-3', reverse primer $5^{\prime}$-CTGTGAATAATTTGCACATTGC-3' (Invitrogen), $\mathrm{MgCl}_{2}, \mathrm{dNTPs}$, Taq polymerase, $\mathrm{H}_{2} \mathrm{O}$, and buffer were added to the DNA. The DNA was amplified by denaturation for $5 \mathrm{~min}$ in $94^{\circ} \mathrm{C}, 30$ cycles of $1 \mathrm{~min}$ denaturation in $94^{\circ} \mathrm{C}, 1 \mathrm{~min}$ annealing in $57^{\circ} \mathrm{C}$ and $30 \mathrm{~s}$ extension in $72^{\circ} \mathrm{C}$, and finally extension for $7 \mathrm{~min}$ in $72^{\circ} \mathrm{C}$. PCR products were digested with BseJL (Fermenta) for $4 \mathrm{~h}$ at $65^{\circ} \mathrm{C}$. CCR2-V64I variants were detected by electrophoreses on $3 \%$ agarose gel.

2.7. Statistics. Statistics and calculations were performed in SPSS version 20. Statistical significance was considered if $p<0.05$. Analyses of the connection between uMCP-1 and other parameters were performed using the nonparametric tests Kruskal-Wallis and Mann-Whitney $U$ test.

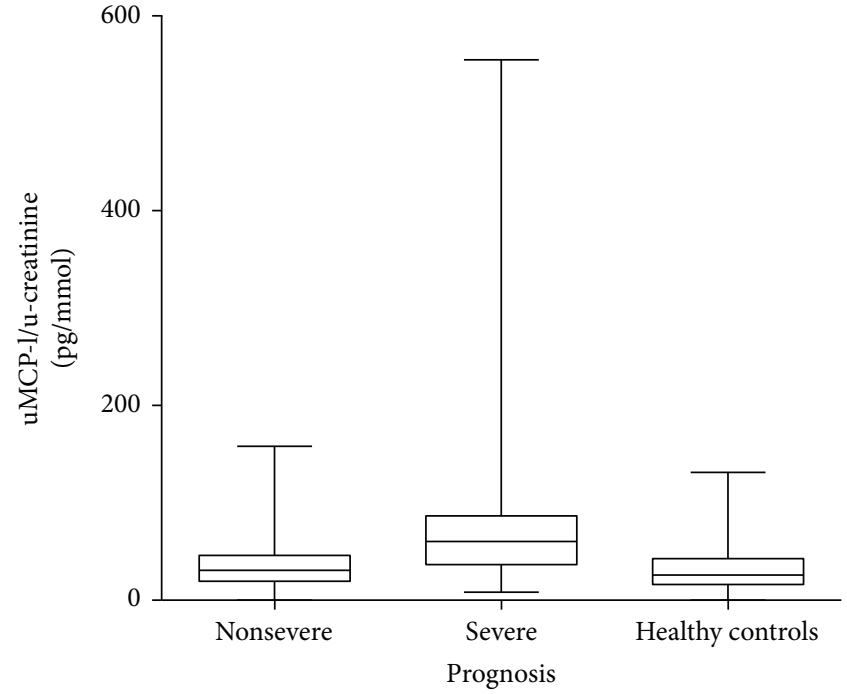

Figure 2: Comparison of uMCP-1 levels in patients with severe prognosis. Nonsevere prognosis and healthy controls. Only urine samples in remission are included. All samples are divided with urinary creatinine levels. uMCP-1: urinary monocyte chemoattractant protein-1. Patients with severe prognosis had higher uMCP-1 levels $(p<0.001)$.

Correlation of uMCP-1 with other parameters was studied using Spearman's rho test. The polymorphism frequencies were studied by the Pearson chi-square test.

\section{Results}

3.1. uMCP-1. All MCP-1 values were divided by u-creatinine before statistical analysis, in order to compensate for the impact of varying urine concentration. Comparison of the mean MCP-1 levels in remission in urine in patients with severe prognosis, nonsevere prognosis, and healthy controls showed significantly higher uMCP-1 values in patients with severe prognosis $(n=46)$ compared to patients with nonsevere prognosis $(n=68, p<0.001)$ and compared to healthy controls $(n=24, p<0.001)$ (see Figure 2$)$. The patients with nonsevere prognosis did not have significantly higher levels of MCP-1 in urine compared to healthy controls. Patients with other vasculitis diagnoses $(n=9)$ had lower levels than the AAV patients with poor prognosis, but higher than the healthy controls (data not shown).

There were 45 patients who scored for renal damage in VDI. Six of these had urine samples taken from both before and after the appearance of kidney damage (see Figures 3 and 4). uMCP-1 seem to peak around the time point of kidney damage. When comparing the mean value in each patient of all the samples taken without/before kidney damage and the mean value of all samples taken with/after kidney damage appeared, uMCP-1 levels were significantly higher when kidney damage had appeared $(p<0.0001)$.

There were 10 patients who had urine samples taken both when in remission and when in the active phase, as demonstrated in Figure 5. Overall, in urine samples taken from patients in remission, the amount of MCP-1was significantly lower than in urine samples taken from patients in the active 


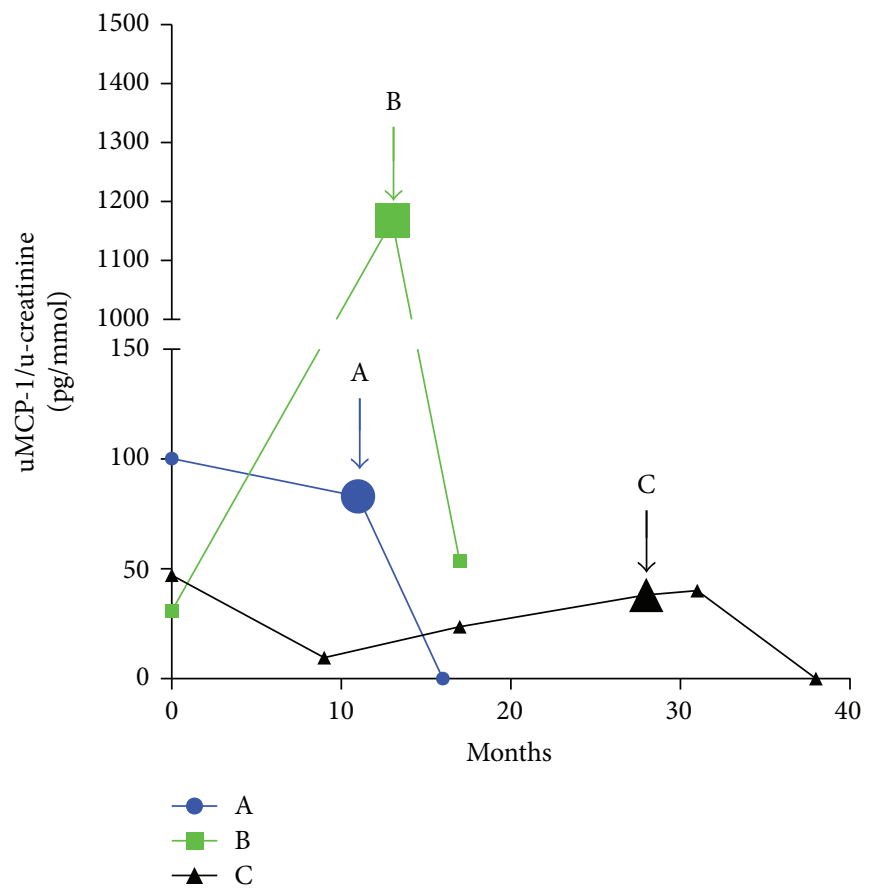

Figure 3: Patients who develop renal damage during the study. Longitudinal view of how uMCP-1 levels changes over time in patients who developed renal damage according to the vasculitis damage index, patients $\mathrm{A}-\mathrm{C}$. The $y$-axis was cut to fit in the high levels of patient $\mathrm{B}$. The arrows point at the first sample taken after renal damage was discovered. Only samples in remission are included. uMCP-1 levels are divided with urinary creatinine levels. uMCP-1: urinary monocyte chemoattractant protein-1.

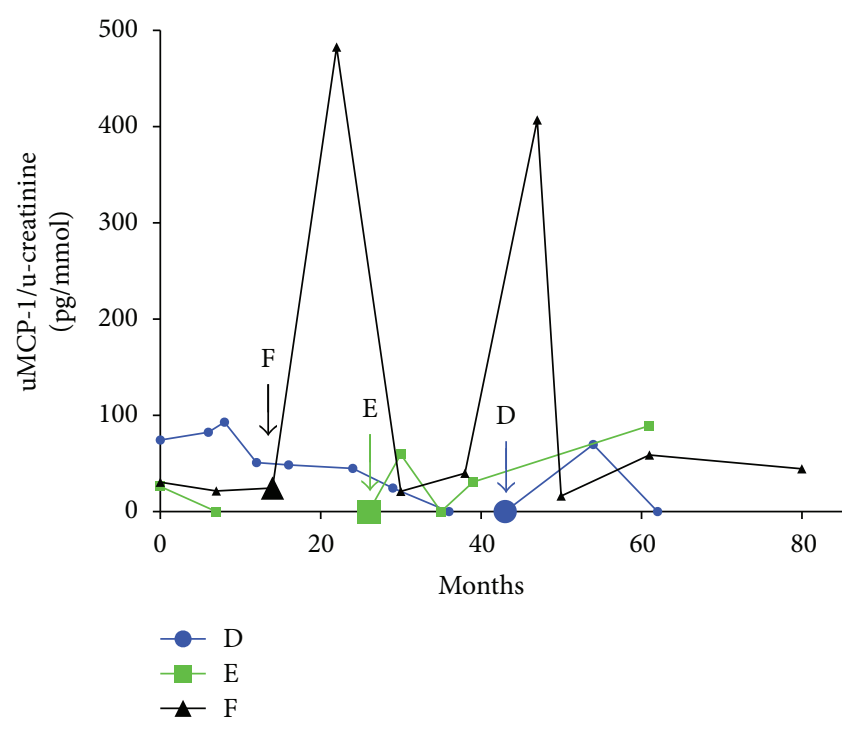

Figure 4: Patients who develop renal damage during the study. Longitudinal view of how uMCP-1 levels changes over time in patients who developed renal damage according to the vasculitis damage index, patients D-F. The $y$-axis was cut to fit in the high levels of patient $\mathrm{B}$. The arrows point at the first sample taken after renal damage was discovered. Only samples in remission are included. uMCP-1 levels are divided with urinary creatinine levels. uMCP-1: urinary monocyte chemoattractant protein-1.

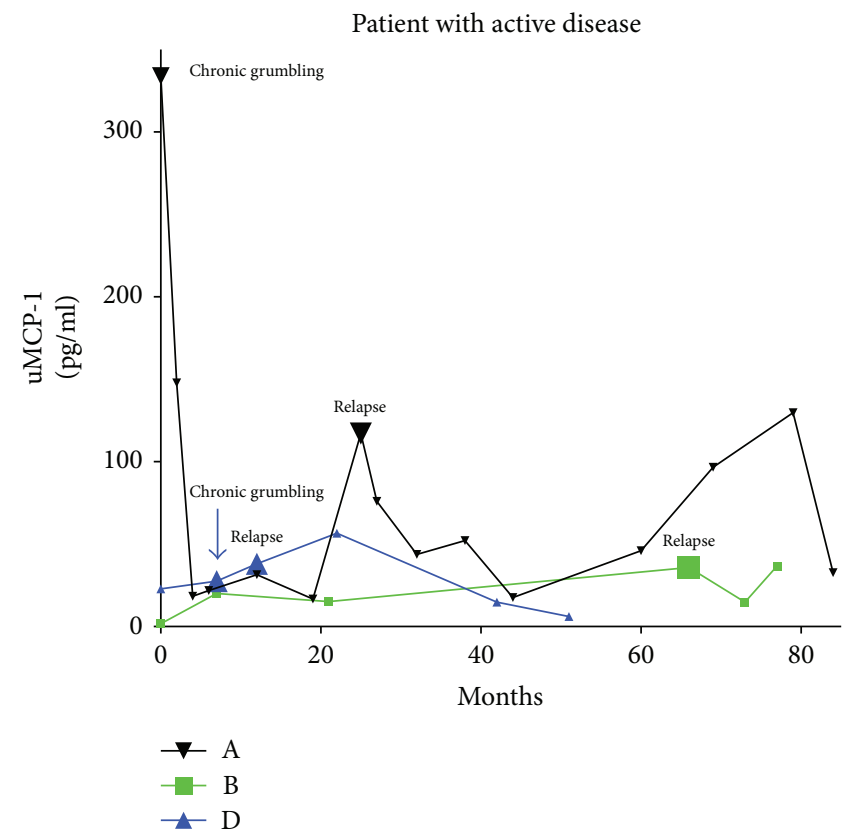

FIgURE 5: uMCP-1 levels in three of the patients who relapsed during the follow-up. All samples are taken in remission except those labelled relapse in the diagram. uMCP-1 levels are divided with urinary creatinine levels. uMCP-1: urinary monocyte chemoattractant protein-1. 


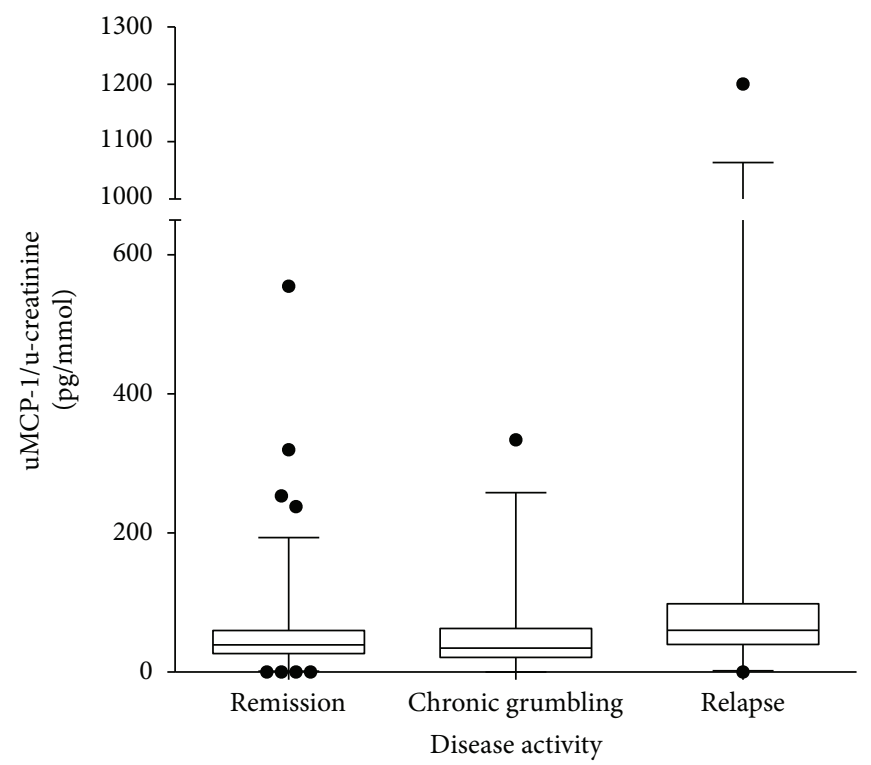

FIGURE 6: Comparison of uMCP-1 levels in different disease activity. uMCP-1 levels are divided with urinary Creatinine levels. The $y$-axis was cut to fit in the high levels of the relapse group. uMCP-1 levels are divided with urinary creatinine levels. uMCP-1: urinary monocyte chemoattractant protein-1. uMCP-1 was higher in relapse than in remission $(p<0.05)$.

phase $(p=0.023)$. Urine samples taken when the patients were in the chronic grumbling phase had neither significantly higher levels of MCP-1 compared to samples taken in remission nor significantly lower levels than patients in the active phase (Figure 6).

There were no significant differences in the amounts of MCP-1 regarding diagnosis or gender. Patients with seronegative AAV had significantly lower uMCP-1 levels compared to patients with MPO-ANCA $(p=0.046)$, and no other significant differences were seen regarding ANCA specificity.

Based on the levels of uMCP-1 over the 3rd quartile in healthy controls, the patients' mean uMCP-1 values were classified as high. Levels of uMCP-1 under the 1st quartile in healthy controls were classified as low. When looking at poor prognosis, the positive predictive value was $70 \%$ and the negative predictive value was $87.5 \%$.

There was a significant correlation between levels of uMCP-1 and levels of CRP, white blood cell count, and creatinine in serum. There was also a significant correlation with U-albumin/creatinine index $(p<0.01)$ (see Table 2).

\subsection{Polymorphism in the MCP-1 Gene and CCR2 Gene. There} were no significant differences between the number of patients having the different genotypes at position -2518 in the MCP-1 gene, as compared to the distribution among the healthy controls (see Figure 7 for more details regarding the polymorphism analysis). Neither was there any difference regarding the CCR2-V64I mutation in patients compared to healthy controls. No differences in the investigated genotypes were seen regarding diagnosis or ANCA specificity. However, in patients with severe prognosis, $87.5 \%$ had the A/A genotype, $12.5 \%$ had the $\mathrm{A} / \mathrm{G}$ genotype, and $0 \%$ had the $\mathrm{G} / \mathrm{G}$ genotype. This was a significant difference compared to patients with nonsevere prognosis, where $39.5 \%$ had the A/A genotype, $59.3 \%$ had the A/G genotype, and $5.2 \%$ had the $\mathrm{G} / \mathrm{G}$ genotype $(p=0.0068)$. All patients who developed end-stage renal disease had the A/A genotype. The mean uMCP-1 level was significantly higher in the patients with the A/A genotype compared to the A/G and the G/G genotype $(p=0.02)$ (see Table 3 ). There was no significant difference in the mean uMCP-1 value in patients when comparing the $\mathrm{A} / \mathrm{A}, \mathrm{A} / \mathrm{G}$, and $\mathrm{G} / \mathrm{G}$ genotype of the CCR2 gene.

\section{Discussion}

In a previous study [21], it was shown that uMCP-1 could be a potential marker of severe prognosis and maybe also a marker of active disease. In this longitudinal study, we wanted to further explore the ability of uMCP-1 to predict active disease and relapses and also to confirm its role as a prognostic marker. In addition, we wanted to explore which role earlier described mutations in the MCP-1 gene and the CCR2 have in AAV.

Our results showed that patients with a more severe prognosis had significantly higher levels of uMCP-1 compared to patients with a better prognosis and healthy controls. This was consistent with previous findings. Patients with active disease had significantly $(p=0.023)$ higher levels compared to patients in remission. Together, these findings suggest a higher level of MCP-1 in urine in active disease compared to when in remission. Chronic grumbling disease did not show significantly higher levels of uMCP-1 compared to remission.

Patients with kidney damage according to VDI had significantly higher uMCP-1 than patients without kidney damage. Patients with kidney damage had in higher extent severe prognosis, compared to patients without kidney damage. The patients with kidney damage correlated well with the patients with poor prognosis (data not shown), and it has been shown 


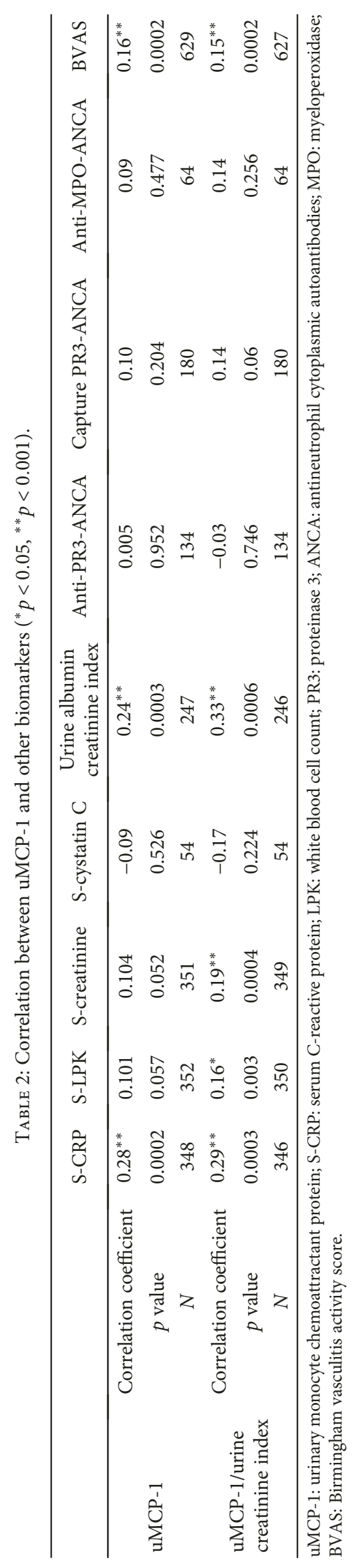




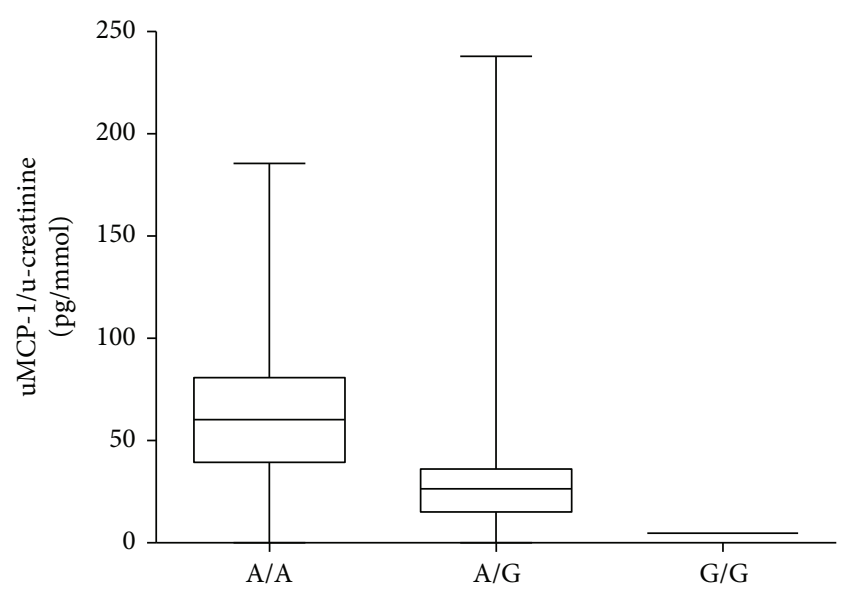

FIGURE 7: Comparison of uMCP-1 levels in patients with different genotype in the MCP-1 gene at position -2518. uMCP-1 levels are divided with urinary creatinine. uMCP-1 levels are divided with urinary creatinine levels. uMCP-1: urinary monocyte chemoattractant protein-1.

in previous studies that kidney damage correlate with a more severe overall outcome [15-17].

In the previous study, it was discussed why higher levels of uMCP-1 is associated with severe prognosis [21]. Two explanations are presented: either UMCP-1 signals constant subclinical disease activity and this ongoing inflammation is harmful over time, causing a more severe course of the disease, or uMCP-1 is a sign of renal damage which in turn is known to be associated with severe prognosis. MCP-1 is produced locally by kidney cells, and it not only recruits macrophages causing indirect damage but it also can directly start a fibrotic response in glomerular mesangial cells [41]. Our previous study showed a correlation with urinary protein HC and S-creatinine, and the correlation with S-creatinine was also seen in this study. These are indicators of renal damage, supporting the explanation of UMCP-1 as a marker of kidney damage. uMCP-1 has been shown to correlate with disease activity and with renal involvement in patients with SLE [24]. Furthermore, uMCP-1 is also associated with renal damage in diabetic nephropathy and it has been concluded that proteinuria increases the MCP-1 expression which accelerates diabetic nephropathy [42]. It is reasonable to believe that this is also the case in AAV and that MCP-1 is involved in the pathogenesis as well as being a biomarker of disease activity and prognosis.

Showing a high negative predictive value, low UMCP-1 values can to a high extent rule out a severe prognosis. This could be a help in adjusting the treatment more individually, perhaps with lower dosages or faster withdrawal of immunosuppressants. The positive predictive value was lower, but at the same time, we saw that patients with the highest values of uMCP-1 solely had severe prognosis (data not shown). This could be a guideline for more aggressive treatment. A proteomics study from 2009 defined a number of biomarkers that were able to distinguish between patients with active AAV and patients in remission. Proteolytic products of hemoglobin were the most frequently observed, but fragments of albumin and a1-antitrypsin were also seen. The study suggested the usefulness of a panel of urinary biomarkers [23]. uMCP-1 could be a good complement to other markers in such a panel. Continued research in the area is needed.

There are some studies on genetic correlations with AAV. In the 2012 European GWAS, AAV was associated not only with MHC but also with a single-nucleotide polymorphism (SNP) in the SERPINA1 locus. The strongest genetic associations were seen with the ANCA specificities; ANCA against PR3 was associated with HLA-DP and the genes encoding for $\alpha 1$-antitrypsine and proteinase 3 . It was also shown that MPA and GPA are genetically distinct [43]. Here, we explored the association of SNPs in the CCR2 gene and the MCP-1 gene. There were no significant differences in the different genotypes in the CCR2 in the context of prognosis or uMCP-1 levels. The A/A genotype at -2518 in the MCP-1 gene was associated with severe prognosis and higher uMCP-1 levels than the A/G and G/G genotype. The SNP is situated in the regulatory region of MCP-1 and has been seen to increase the expression of MCP-1 in healthy individuals. The SNP has been shown to be associated with various diseases, like SLE [27, 44], systemic sclerosis [45], pulmonary tuberculosis [46], psoriasis [47], kidney transplant outcome [48], type 1 diabetes [49], renal disease progression in IgA nephritis [50], kidney failure in patients with diabetes mellitus type 2 [51], lupus nephritis [27], and severity in Behcet's disease [28]. The studies mentioned show somewhat confusing results, associating either the $A$ allele or the $G$ allele with risk of disease and increased MCP-1 expression. The cause of these divergent results is obscure. As mentioned before, our group demonstrated a deviant profile of cytokines in patients with AAV compared to healthy individuals [21, 52], and disease-specific conditions like this could play a role. The genetic differences between the populations in the different studies are another factor to consider. There are two metaanalyses from 2016. Lee and Bae gathered in total 14 publications and 3038 patients suffering from vasculitis, RA, or MS and found no significant association with the $G$ allele [53]. On the other hand, Chen et al. associates the A allele with an increased risk of autoimmunity in their meta-analysis of studies on RA, Crohn's disease, and lupus nephritis [54]. The number of patients in the present study is rather small, and a larger study is needed to further investigate the association between MCP-1 polymorphism and AAV prognosis. There is also a need for further studies of the local production of MCP-1 in the kidney and migration of monocytes/macrophages to the renal tissue in relation to the MCP-1 -2518 A/G polymorphism.

\section{Summary}

In conclusion, MCP-1 levels in urine can be useful in determining if a patient is prone to have a more severe outcome. MCP-1 levels are significantly higher in patients with active disease and in patients with kidney damage. Polymorphism -2518A homozygous is associated with a more severe outcome and higher levels of uMCP-1, whereas CCR2-V64I did not show such a correlation. Further research is needed in the field. 


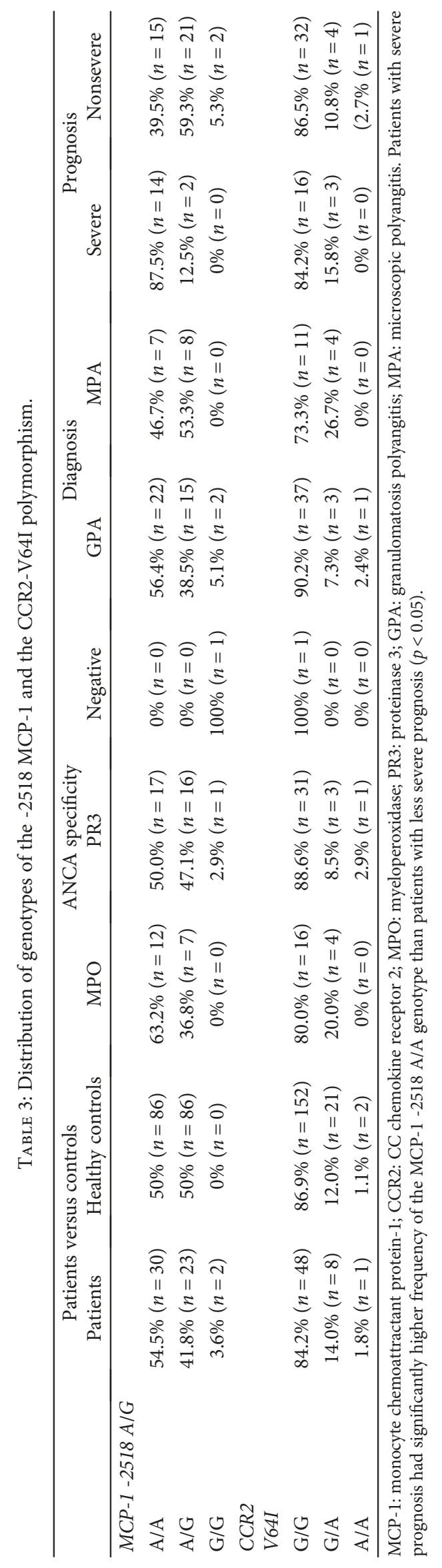




\section{Disclosure}

Some of the data presented in this article were included in a poster presentation in 2013 at the 16th International Vasculitis and ANCA Workshop, Paris.

\section{Conflicts of Interest}

The authors declare that there is no conflict of interest regarding the publication of this paper.

\section{References}

[1] D. J. Davies, J. E. Moran, J. F. Niall, and G. B. Ryan, "Segmental necrotising glomerulonephritis with antineutrophil antibody: possible arbovirus aetiology?," BMJ, vol. 285, no. 6342, p. 606, 1982

[2] P. Lesavre, "Antineutrophil cytoplasmic autoantibodies antigen specificity," American Journal of Kidney Diseases, vol. 18, no. 2, pp. 159-163, 1991.

[3] R. J. Falk and J. C. Jennette, "Anti-neutrophil cytoplasmic autoantibodies with specificity for myeloperoxidase in patients with systemic vasculitis and idiopathic necrotizing and crescentic glomerulonephritis," New England Journal of Medicine, vol. 318, no. 25, pp. 1651-1657, 1988.

[4] F. J. van der Woude, N. Rasmussen, S. Lobatto et al., "Autoantibodies against neutrophils and monocytes: tool for diagnosis and marker of disease activity in Wegener's granulomatosis," Lancet, vol. 1, no. 8426, pp. 425-429, 1985.

[5] J. C. Jennette and R. J. Falk, "Small-vessel vasculitis," The New England Journal of Medicine, vol. 337, no. 21, pp. 1512-1523, 1997.

[6] J. H. Stone, "Limited versus severe Wegener's granulomatosis: baseline data on patients in the Wegener's granulomatosis etanercept trial," Arthritis \& Rheumatism, vol. 48, no. 8, pp. 2299-2309, 2003.

[7] B. H. Ewert, J. C. Jennette, and R. J. Falk, “Anti-myeloperoxidase antibodies stimulate neutrophils to damage human endothelial cells," Kidney International, vol. 41, no. 2, pp. 375-383, 1992.

[8] A. Wikman, A. Fagergren, S. Gunnar, O. Johansson, J. Lundahl, and S. H. Jacobson, "Monocyte activation and relationship to anti-proteinase 3 in acute vasculitis," Nephrology Dialysis Transplantation, vol. 18, no. 9, pp. 1792-1799, 2003.

[9] C. F. M. Franssen, C. A. Stegeman, C. G. M. Kallenberg et al., "Antiproteinase 3- and antimyeloperoxidase-associated vasculitis," Kidney International, vol. 57, no. 6, pp. 2195-2206, 2000.

[10] A. J. Mohammad, L. T. H. Jacobsson, A. D. Mahr, G. Sturfelt, and M. Segelmark, "Prevalence of Wegener's granulomatosis, microscopic polyangiitis, polyarteritis nodosa and ChurgStrauss syndrome within a defined population in southern Sweden," Rheumatology, vol. 46, no. 8, pp. 1329-1337, 2007.

[11] C. Mukhtyar, O. Flossmann, B. Hellmich et al., "Outcomes from studies of antineutrophil cytoplasm antibody associated vasculitis: a systematic review by the European Leage Against Rheumatism systemic vasculitis task force," Annals of the Rheumatic Diseases, vol. 67, no. 7, pp. 1004-1010, 2008.

[12] C. Mukhtyar, L. Guillevin, M. C. Cid et al., "EULAR recommendations for the management of primary small and medium vessel vasculitis," Annals of the Rheumatic Diseases, vol. 68 , no. 3, pp. 310-317, 2009.
[13] E. Reinhold-Keller, N. Beuge, U. Latza et al., "An interdisciplinary approach to the care of patients with Wegener's granulomatosis: long-term outcome in 155 patients," Arthritis and Rheumatism, vol. 43, no. 5, pp. 1021-1032, 2000.

[14] J. U. Holle, W. L. Gross, U. Latza et al., "Improved outcome in 445 patients with Wegener's granulomatosis in a German vasculitis center over four decades," Arthritis \& Rheumatism, vol. 63, no. 1, pp. 257-266, 2011.

[15] H. A. Hauer, I. M. Bajema, H. C. Van Houwelingen et al., "Determinants of outcome in ANCA-associated glomerulonephritis: a prospective clinico-histopathological analysis of 96 patients," Kidney International, vol. 62, no. 5, pp. 1732-1742, 2002.

[16] M. C. Slot, J. W. C. Tervaert, C. F. M. Franssen, and C. A. Stegeman, "Renal survival and prognostic factors in patients with PR3-ANCA associated vasculitis with renal involvement," Kidney International, vol. 63, no. 2, pp. 670-677, 2003.

[17] C. F. Franssen, C. A. Stegeman, W. W. Oost-Kort et al., "Determinants of renal outcome in anti-myeloperoxidase-associated necrotizing crescentic glomerulonephritis," Journal of the American Society of Nephrology, vol. 9, no. 10, pp. 19151923, 1998.

[18] P. Cockwell, C. J. Brooks, D. Adu, and C. O. S. Savage, "Interleukin-8: a pathogenetic role in antineutrophil cytoplasmic autoantibody-associated glomerulonephritis," Kidney International, vol. 55, no. 3, pp. 852-863, 1999.

[19] O. Bakoush, M. Segelmark, O. Torffvit, S. Ohlsson, and J. Tencer, "Urine IgM excretion predicts outcome in ANCAassociated renal vasculitis," Nephrology, Dialysis, Transplantation, vol. 21, no. 5, pp. 1263-1269, 2006.

[20] K. W. A. Westman, D. Selga, P.-E. Isberg, A. Bladström, and H. Olsson, "High proteinase 3-anti-neutrophil cytoplasmic antibody (ANCA) level measured by the capture enzymelinked immunosorbent assay method is associated with decreased patient survival in ANCA-associated vasculitis with renal involvement," Journal of the American Society of Nephrology, vol. 14, no. 11, pp. 2926-2933, 2003.

[21] S. Ohlsson, O. Bakoush, J. Tencer, O. Torffvit, and M. Segelmark, "Monocyte chemoattractant protein 1 is a prognostic marker in ANCA-associated small vessel vasculitis," Mediators of Inflammation, vol. 2009, Article ID 584916, 7 pages, 2009.

[22] M. M. Boomsma, C. A. Stegeman, M. J. Van Der Leij et al., "Prediction of relapses in Wegener's granulomatosis by measurement of antineutrophil cytoplasmic antibody levels: a prospective study," Arthritis \& Rheumatism, vol. 43, no. 9, pp. 2025-2033, 2000.

[23] M. Haubitz, D. M. Good, A. Woywodt et al., "Identification and validation of urinary biomarkers for differential diagnosis and evaluation of therapeutic intervention in anti-neutrophil cytoplasmic antibody-associated vasculitis," Molecular \& Cellular Proteomics, vol. 8, no. 10, pp. 2296-2307, 2009.

[24] R. Camilla, S. Brachemi, V. Pichette et al., "Urinary monocyte chemotactic protein 1: marker of renal function decline in diabetic and nondiabetic proteinuric renal disease," Journal of Nephrology, vol. 24, no. 1, pp. 60-67, 2011.

[25] R. Herbst, Z. Liu, B. Jallal, and Y. Yao, "Biomarkers for systemic lupus erythematosus," International Journal of Rheumatic Diseases, vol. 15, no. 5, pp. 433-444, 2012.

[26] B. H. Rovin, L. Lu, and R. Saxena, "A novel polymorphism in the MCP-1 gene regulatory region that influences MCP-1 
expression," Biochemical and Biophysical Research Communications, vol. 259, no. 2, pp. 344-348, 1999.

[27] H. L. Kim, D. S. Lee, S. H. Yang et al., "The polymorphism of monocyte chemoattractant protein-1 is associated with the renal disease of SLE," American Journal of Kidney Diseases, vol. 40, no. 6, pp. 1146-1152, 2002.

[28] S. K. Kim, W. C. Jang, Y. C. Ahn, S. H. Lee, S. S. Lee, and J. W. Hur, "Promoter -2518 single nucleotide polymorphism of monocyte chemoattractant protein-1 is associated with clinical severity in Behçet's disease," Inflammation Research, vol. 61, no. 6, pp. 541-545, 2012.

[29] J. Bai, H. Song, C. Cai, M. Zhang, S. Xu, and J. Tan, “The association of monocyte chemotactic protein-1 and CC chemokine receptor 2 gene variants with chronic obstructive pulmonary disease," DNA and Cell Biology, vol. 31, no. 6, pp. 1058-1063, 2012.

[30] K. Bektas-Kayhan, M. Unur, Z. Boy-Metin, and B. Cakmakoglu, "MCP-1 and CCR2 gene variants in oral squamous cell carcinoma," Oral Diseases, vol. 18, no. 1, pp. 55-59, 2012.

[31] S. W. Kang, S. J. Park, Y. W. Kim et al., "Association of MCP-1 and CCR2 polymorphisms with the risk of late acute rejection after renal transplantation in Korean patients," International Journal of Immunogenetics, vol. 35, no. 1, pp. 25-31, 2008.

[32] S. L. Deshmane, S. Kremlev, S. Amini, and B. E. Sawaya, "Monocyte chemoattractant protein-1 (MCP-1): an overview," Journal of Interferon \& Cytokine Research, vol. 29, no. 6, pp. 313-326, 2009.

[33] E. E. Nakayama, Y. Tanaka, Y. Nagai, A. Iwamoto, and T. Shioda, "A CCR2-V64I polymorphism affects stability of CCR2A isoform,” AIDS, vol. 18, no. 5, pp. 729-738, 2004.

[34] R. Miyagishi, M. Niino, T. Fukazawa, I. Yabe, S. Kikuchi, and $\mathrm{K}$. Tashiro, " $\mathrm{C}-\mathrm{C}$ chemokine receptor 2 gene polymorphism in Japanese patients with multiple sclerosis," Journal of Neuroimmunology, vol. 145, no. 1-2, pp. 135-138, 2003.

[35] J. P. Ioannidis, P. S. Rosenberg, J. J. Goedert et al., "Effects of CCR5- $\triangle 32$, CCR2-64I, and SDF-1 3'A alleles on HIV-1 disease progression: an international meta-analysis of individualpatient data," Annals of Internal Medicine, vol. 135, no. 9, pp. 782-795, 2001.

[36] A. Zafiropoulos, N. Crikas, A. M. Passam, and D. A. Spandidos, "Significant involvement of CCR2-64I and CXCL12-3a in the development of sporadic breast cancer," Journal of Medical Genetics, vol. 41, no. 5, article e59, 2004.

[37] R. Watts, S. Lane, T. Hanslik et al., "Development and validation of a consensus methodology for the classification of the ANCA-associated vasculitides and polyarteritis nodosa for epidemiological studies," Annals of the Rheumatic Diseases, vol. 66, no. 2, pp. 222-227, 2007.

[38] R. A. Luqmani, P. A. Bacon, R. J. Moots et al., "Birmingham vasculitis activity score (BVAS) in systemic necrotizing vasculitis," QJM, vol. 87, no. 11, pp. 671-678, 1994.

[39] A. R. Exley, P. A. Bacon, R. A. Luqmani et al., "Development and initial validation of the vasculitis damage index for the standardized clinical assessment of damage in the systemic vasculitides," Arthritis \& Rheumatism, vol. 40, no. 2, pp. 371-380, 1997.

[40] C. J. Beall, S. Mahajan, D. E. Kuhn, and P. E. Kolattukudy, "Site-directed mutagenesis of monocyte chemoattractant protein-1 identifies two regions of the polypeptide essential for biological activity," The Biochemical Journal, vol. 313, no. 2, pp. 633-640, 1996.

[41] G. H. Tesch, "MCP-1/CCL2: a new diagnostic marker and therapeutic target for progressive renal injury in diabetic nephropathy," American Journal of Physiology-Renal Physiology, vol. 294, no. 4, pp. F697-F701, 2008.

[42] T. Morii, H. Fujita, T. Narita et al., "Association of monocyte chemoattractant protein-1 with renal tubular damage in diabetic nephropathy," Journal of Diabetes and its Complications, vol. 17, no. 1, pp. 11-15, 2003.

[43] P. A. Lyons, T. F. Rayner, S. Trivedi et al., "Genetically distinct subsets within ANCA-associated vasculitis," The New England Journal of Medicine, vol. 367, no. 3, pp. 214-223, 2012.

[44] M. Tucci, E. V. Barnes, E. S. Sobel et al., "Strong association of a functional polymorphism in the monocyte chemoattractant protein 1 promoter gene with lupus nephritis," Arthritis \& Rheumatism, vol. 50, no. 6, pp. 1842-1849, 2004.

[45] S. Karrer, A. K. Bosserhoff, P. Weiderer et al., "The -2518 promotor polymorphism in the MCP-1 gene is associated with systemic sclerosis," The Journal of Investigative Dermatology, vol. 124, no. 1, pp. 92-98, 2005.

[46] P. O. Flores-Villanueva, J. A. Ruiz-Morales, C. H. Song et al., "A functional promoter polymorphism in monocyte chemoattractant protein-1 is associated with increased susceptibility to pulmonary tuberculosis," The Journal of Experimental Medicine, vol. 202, no. 12, pp. 1649-1658, 2005.

[47] L. Wang, L. Yang, L. Gao, T. W. Gao, W. Li, and Y. F. Liu, “A functional promoter polymorphism in monocyte chemoattractant protein-1 is associated with psoriasis," International Journal of Immunogenetics, vol. 35, no. 1, pp. 45-49, 2008.

[48] B. Krüger, B. Schröppel, R. Ashkan et al., “A monocyte chemoattractant protein-1 (MCP-1) polymorphism and outcome after renal transplantation," Journal of the American Society of Nephrology, vol. 13, no. 10, pp. 2585-2589, 2002.

[49] B. Yang, K. Houlberg, A. Millward, and A. Demaine, "Polymorphisms of chemokine and chemokine receptor genes in type 1 diabetes mellitus and its complications," Cytokine, vol. 26, no. 3, pp. 114-121, 2004.

[50] H. Mori, Y. Kaneko, I. Narita et al., "Monocyte chemoattractant protein-1 A-2518G gene polymorphism and renal survival of Japanese patients with immunoglobulin A nephropathy," Clinical and Experimental Nephrology, vol. 9, no. 4, pp. 297-303, 2005.

[51] J. Y. Moon, L. Jeong, S. Lee et al., “Association of polymorphisms in monocyte chemoattractant protein-1 promoter with diabetic kidney failure in Korean patients with type 2 diabetes mellitus," Journal of Korean Medical Science, vol. 22, no. 5, pp. 810-814, 2007.

[52] S. Ohlsson, J. Wieslander, and M. Segelmark, "Circulating cytokine profile in anti-neutrophilic cytoplasmatic autoantibodyassociated vasculitis: prediction of outcome?," Mediators of Inflammation, vol. 13, no. 4, pp. 275-283, 2004.

[53] Y. H. Lee and S. C. Bae, "Monocyte chemoattractant protein-1 promoter -2518 polymorphism and susceptibility to vasculitis, rheumatoid arthritis, and multiple sclerosis: a meta-analysis," Cellular and Molecular Biology, vol. 62, no. 3, pp. 65-71, 2016.

[54] S. Chen, C. Deng, C. Hu et al., "Association of MCP-1-2518A/ $\mathrm{G}$ polymorphism with susceptibility to autoimmune diseases: a meta-analysis," Clinical Rheumatology, vol. 35, no. 5, pp. 1169-1179, 2016. 


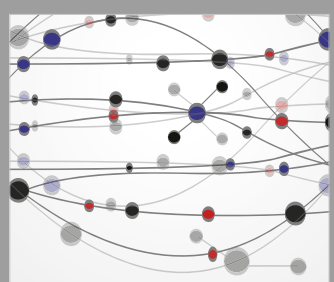

The Scientific World Journal
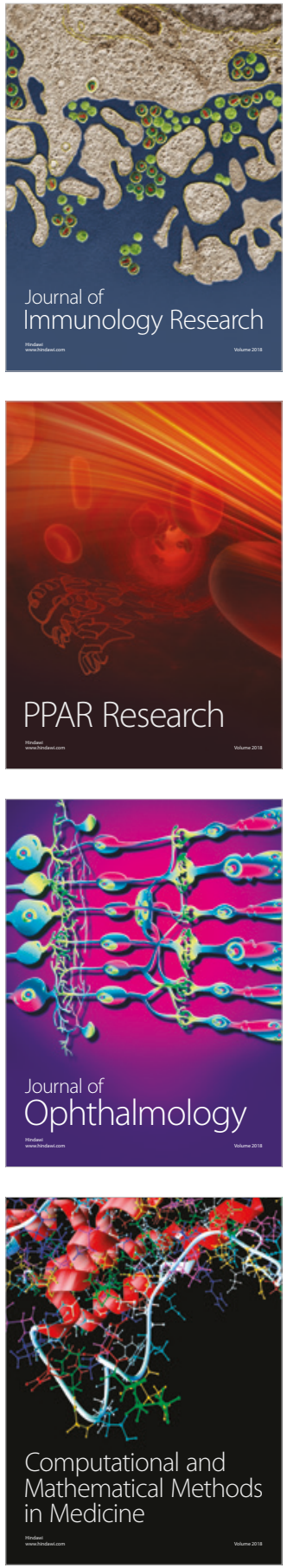

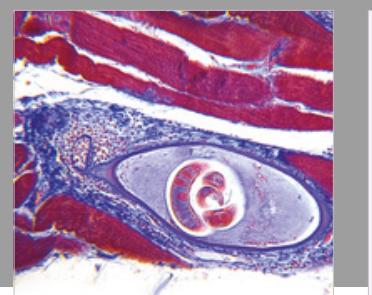

Gastroenterology Research and Practice

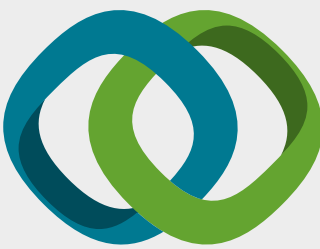

\section{Hindawi}

Submit your manuscripts at

www.hindawi.com
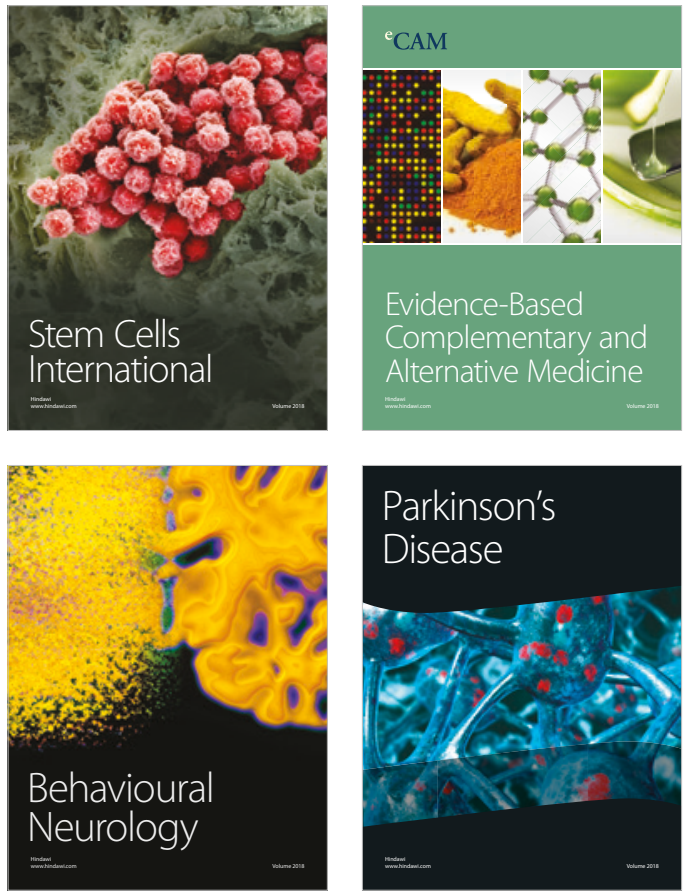

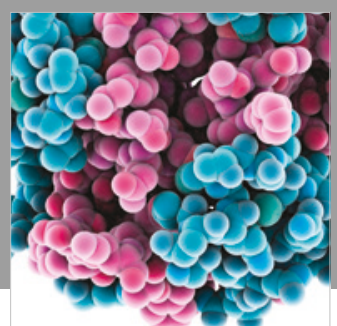

ournal of

Diabetes Research

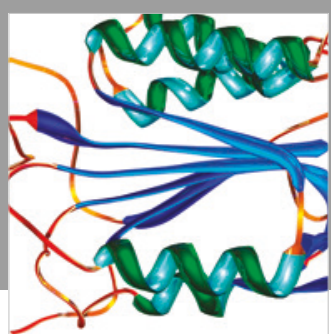

Disease Markers
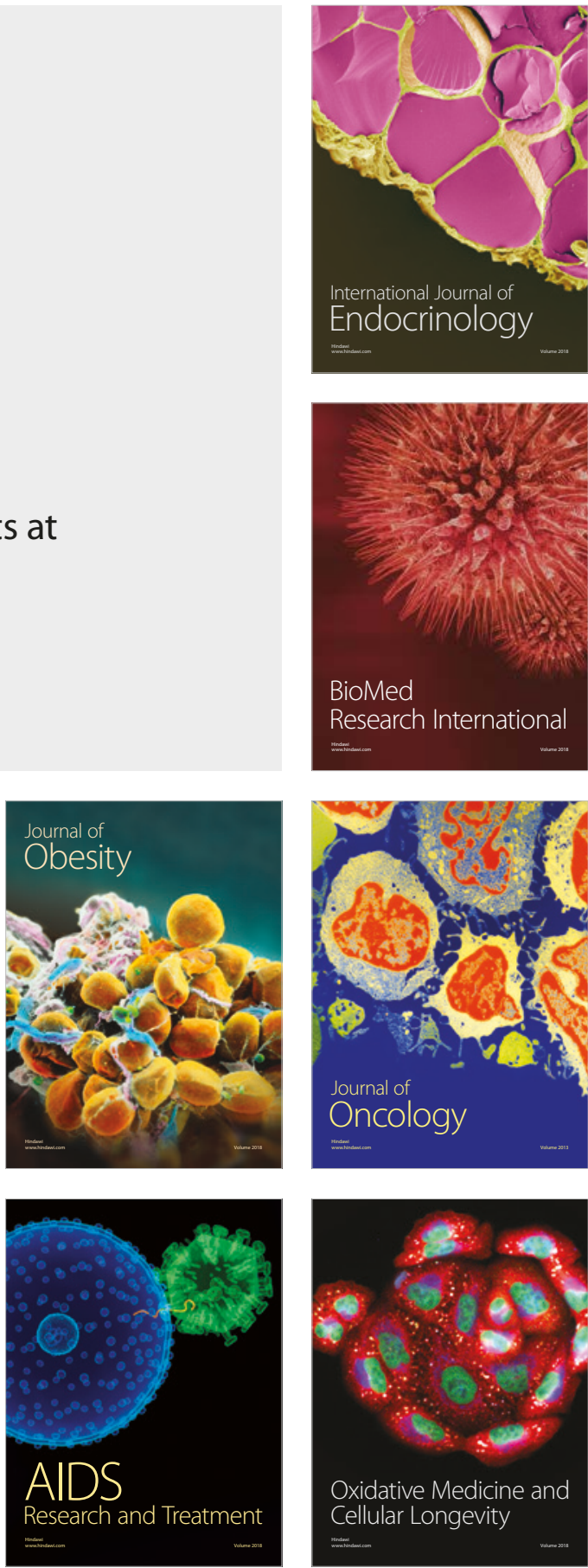